First Peoples Child \& Family Review

An Interdisciplinary Journal Honouring the Voices, Perspectives, and Knowledges of

First Peoples through Research, Critical Analyses, Stories, Standpoints and Media

Reviews

\title{
Le dépistage des retards de développement chez les jeunes enfants d'une communauté des Premières Nations
}

\section{Carmen Dionne, Suzie McKinnon and Jane Squires}

Volume 5, Number 2, 2010

URI: https://id.erudit.org/iderudit/1068937ar

DOI: https://doi.org/10.7202/1068937ar

See table of contents

Publisher(s)

First Nations Child and Family Caring Society of Canada

\section{ISSN}

1708-489X (print)

2293-6610 (digital)

Explore this journal

Cite this article

Dionne, C., McKinnon, S. \& Squires, J. (2010). Le dépistage des retards de développement chez les jeunes enfants d'une communauté des Premières Nations. First Peoples Child \& Family Review, 5(2), 117-123.

https://doi.org/10.7202/1068937ar
Article abstract

Bien qu'il importe d'utiliser des instruments d'évaluation adaptés aux caractéristiques et à la réalité des communautés autochtones, l'absence de normes psychométriques pour ces communautés est fréquemment déplorée. Le but est de documenter la pertinence et l'utilité de l'instrument de dépistage des retards de développement Age and Stages Questionnaires (ASQ) utilisé par des enseignantes auprès d'enfants $(n=213)$ Mohawks du Québec âgés entre 29 et 60 mois. Au niveau de la consistance interne pour les quatre questionnaires étudiés, sept résultats révèlent des alphas de Cronbach inférieurs à 0,60 : « communication " (Q42), « motricité globale " (Q36 et Q42), " motricité fine " (Q42) et « aptitudes individuelles ou sociales » (Q36, Q42 et Q54) tandis que treize présentent des alphas de Cronbach variant entre 0,61 et 0,90.

Globalement, le groupe de discussion réalisé auprès des enseignantes révèle leur appréciation de l'instrument et le fait que son utilisation apparaît approprié pour cette communauté.
Copyright (c) Carmen Dionne, Suzie McKinnon, Jane Squires, 2010

This document is protected by copyright law. Use of the services of Érudit (including reproduction) is subject to its terms and conditions, which can be viewed online.

https://apropos.erudit.org/en/users/policy-on-use/ 


\title{
First Peoples Child \& Family Review
}

An Interdisciplinary Journal Honoring the Voices, Perspectives and Knowledges of First Peoples through Research, Critical Analyses, Stories, Standpoints and Media Reviews

\section{Le dépistage des retards de développement chez les jeunes enfants d'une communauté des Premières Nations}

\author{
Carmen Dionne ${ }^{\mathrm{a}}$, Suzie McKinnon ${ }^{\mathrm{b}}$ and Jane Squires ${ }^{\mathrm{c}}$
}

\begin{abstract}
a Ph.D., Titulaire de la Chaire de recherche du Canada en intervention précoce, Professeure-chercheure au Département de psychoéducation, Université du Québec à Trois-Rivières, CP 500, Trois-Rivières, G9A 5H7, 819-376-5011 \# 4008 (phone), 376-5066 (fax), carmen.dionne@uqtr.ca

${ }^{b}$ Ph.D., Département de psychoéducation, Université du Québec à TroisRivières.

${ }^{\mathrm{c}}$ Ph.D., Early Intervention Program, University of Oregon.
\end{abstract}

\section{Introduction}

Les toutes premières années de vie des jeunes enfants sont des périodes majeures de développement. Le cerveau présente alors une plasticité maximale permettant aux stimulations de lenvironnement d'exercer une influence prépondérante sur son organisation (notamment Shonkoff \& Philips, 2000). Pour les enfants présentant des besoins particuliers, l'importance de débuter l'intervention spécialisée le plus tôt possible fait l'objet d'un très vaste consensus.

Afin de sassurer que les interventions soient les plus appropriées aux besoins de ces enfants, le dépistage des difficultés ou retards de développement s'impose avec acuité. Cependant, 65\% des enfants ayant des besoins spéciaux n'ont pas reçu de diagnostic (CSSSPNQL, 2008). Trop peu d'outils de dépistage, ayant une solide validité et fidélité et pouvant

Questions or correspondence concerning this article may be addressed to:

Carmen Dionne

Chaire de recherche du Canada en intervention précoce

Département de psychoéducation

Université du Québec à Trois-Rivières, CP 500

Trois-Rivières, Québec, G9A 5H7 Canada

819.376.5011 \# 4008 (phone)

819.376.5066 (fax)

carmen.dionne@uqtr.ca

\section{Résumé de l'article}

Bien qu'il importe d'utiliser des instruments d'évaluation adaptés aux caractéristiques et à la réalité des communautés autochtones, l'absence de normes psychométriques pour ces communautés est fréquemment déplorée. Le but est de documenter la pertinence et l'utilité de l'instrument de dépistage des retards de développement Age and Stages Questionnaires (ASQ) utilisé par des enseignantes auprès d'enfants $(n=213)$ Mohawks du Québec âgés entre 29 et 60 mois. Au niveau de la consistance interne pour les quatre questionnaires étudiés, sept résultats révèlent des alphas de Cronbach inférieurs à 0,60 : " communication » (Q42), « motricité globale » (Q36 et Q42), « motricité fine » (Q42) et « aptitudes individuelles ou sociales » (Q36, Q42 et Q54) tandis que treize présentent des alphas de Cronbach variant entre 0,61 et 0,90 . Globalement, le groupe de discussion réalisé auprès des enseignantes révèle leur appréciation de l'instrument et le fait que son utilisation apparaît approprié pour cette communauté.

Mots clés: children, Mohawks, development, developmental delay, screening, psychometric, instrument, early identification.

être utilisé par différents types d'intervenants, sont disponibles. De plus, une certaine confusion existe entre les évaluations du développement des enfants utiles à des fins de diagnostic et celles utiles pour l'intervention (Losardo \& Notari-Syverson, 2001). D'ailleurs, trop souvent des instruments diagnostiques sont utilisés à des fins de dépistage mobilisant ainsi des ressources importantes.

Comme mis en évidence par Meisels (1994), la finalité d'une évaluation de dépistage est de vérifier si l'enfant a besoin d'une évaluation plus spécifique. Le but principal est donc de vérifier la pertinence de référer lenfant à un professionnel pour que ce dernier détermine la nature des difficultés présentées par l'enfant. Ce type dévaluation doit être utilisé lorsque la difficulté n’est pas encore documentée. Les évaluations diagnostiques, quant à elles, regroupent un ensemble d'items choisis de façon 


\section{Acknowledgements:}

The authors wish to thank the parents and staff of Step by Step Child and Family Center for their invaluable collaboration on this study. We wish also to mention the financial support of the Social Sciences and Humanities Research Council (SSHRC).

statistique afin de comparer la performance d'un enfant à un échantillon normatif. Ainsi, ces items ne sont pas nécessairement des habiletés fonctionnelles. Autrement dit, les comportements sélectionnés ne sont pas forcément des comportements utilisés dans la vie quotidienne. Il s'agit d'une caractéristique essentielle qui distingue ce genre d'instruments, des évaluations utiles à des fins d'intervention.

Le recours à des outils pour le réaliser de dépistage s'impose. Ainsi, $30 \%$ des difficultés de développement peuvent être identifiés (Palfrey, Singer, Walker, \& Butler, 1987) alors que ce taux augmente à 70-80\% lorsque des instruments utiles à cette fin sont utilisés (Squires, Nickel, \& Eisert, 1996).

En ce qui concerne les peuples autochtones, il existe peu d'études en petite enfance portant spécifiquement sur cette population (Palmantier, 2005). Selon Hernandez (1994), la plupart des tests standardisés ne sont pas développés en tenant compte des diversités culturelles. Plusieurs parents et intervenants autochtones en petite enfance croient également que les outils formels pour soutenir les enfants et les familles non autochtones ne sont ni culturellement appropriés ni aidant pour leurs enfants (BC Aboriginal Child Care Society 2003; Royal Commission on Aboriginal peoples, 1996). En effet, le recours à des évaluations non culturellement appropriées peuvent avoir des conséquences négatives sérieuses pour les enfants autochtones telles quel'identification erronée de problématiques et les impacts à long terme de ces erreurs diagnostiques (BC Aboriginal Network for disabilities Society, 1996 in Ball, 2010; Mushquash \& Bova, 2007). Pour ces raisons, il importe que les méthodes utilisées pour la sélection des items, le processus de validation et le processus de standardisation soient considérés. Ball (2006) souligne que la standardisation des instruments devrait minimalement inclure une représentativité des populations autochtones au niveau de l'échantillon.

Dans son étude sur les programmes en intervention précoce chez les autochtones de l'Ouest canadien, les résultats de Ball (2006) montrent que l'Ages and Stages Questionnaires (ASQ) (Bricker \& Squires, 1999) est l'outil le plus utilisé. Quant à l'étude effectuée pour « The maternal and child health program First Nations and Inuit health branch » par DionStout et Jodoin (2006), elle recommandait l'utilisation de l'ASQ (Bricker \& Squires, 1999) en tant qu'instrument culturellement approprié à la réalité des Premières Nations. Cet instrument est largement utilisé par les Premières Nations en Colombie-
Britannique. Cependant, aucune norme pour les populations autochtones canadiennes n'est disponible.

Cette prise en considération de la dimension culturelle est pourtant une caractéristique des pratiques exemplaires en intervention précoce (Sandall, Hemmeter, Smith, \& McLean, 2005). On souligne que les intervenants doivent être conscients des biais culturels possibles. Il est suggéré que les intervenants, lorsque la situation est appropriée, choisissent seulement des instruments qui ont été développés, validés et standardisés et dont les normes sont établies auprès des enfants présentant les mêmes caractéristiques que ceux évalués. Cette préoccupation trouve dailleurs écho au niveau de la formation des étudiants. Toujours selon Sandall et al. (2005), il est recommandé que les étudiants et le personnel participent à des activités dans lesquelles ils apprennent à développer et à implanter des stratégies d'intervention congruentes et respectueuses des croyances, des valeurs et des traditions aussi bien que la langue dominante et préférée des familles de cultures et de groupes linguistiques différents.

Pour ces raisons, des travaux visant la poursuite de la validation d'instruments de mesure en contexte canadien sont essentiels afin de s’assurer de disposer d'outils adaptés, valides et standardisés pour tous les enfants canadiens tout en considérant les particularités culturelles et linguistiques de différentes communautés.

\section{But}

Dans la présente étude, l'ASQ (Bricker \& Squires, 1999) est utilisé auprès des jeunes enfants du Step By Step Child and Family Center situé dans une communauté des Premières Nations de l'est canadien. Le but est de documenter la pertinence et l'utilité de l'ASQ utilisé par des intervenants auprès d'enfants Mohawks et de recueillir des données pour sa validation dans un tel contexte.

\section{Méthode}

\section{Participants}

Les participants sont les enseignantes de 213 enfants Mohawks (105 filles et 108 garçons), âgés entre 29 et 60 mois, qui fréquentent le Step by Step Child and Family Center de Kahnawake au Québec. Douze d'entre elles ont été sélectionnées sur la base de leur expérience d'utilisation de l'ASQ pour participer au groupe de discussion. 
Des données sociodémographiques sur les familles ont été recueillies pour 165 enfants. La majorité des familles parle l'anglais à la maison. Presque tous les parents sont d'origine Mohawk. Le taux d'obtention d'un diplôme d'études secondaires est de 20,8\% chez les mères. Ce taux est plus élevé chez les pères avec $28,8 \%$ de diplômation. Au niveau collégial, $4,4 \%$ des mères et 3,2 \% des pères ont un diplôme. L'obtention d'un diplôme universitaire est plus élevée chez les mères avec un taux de 7,5\% comparativement à $1,3 \%$ chez les pères. Concernant le revenu familial, $38,9 \%$ des familles participantes ont un revenu familial annuel de moins de $25000 \$$ et que $25,5 \%$ des familles ont un revenu entre $25000 \$$ et $40000 \$$. Finalement, plus du tiers des familles $(35,7 \%)$ ont un revenu de $40000 \$$ et plus annuellement.

\section{Instruments}

\section{Ages and Stages Questionnaires}

L'ASQ (Bricker \& Squires, 1999) est un outil qui évalue le développement de lenfant. Il s'adresse aux parents et aux éducatrices. La deuxième édition de l'outil, utilisée dans la présente recherche, est constituée de 19 questionnaires selon les âges (en mois) suivants : 4, 6, 8, 10, 12, 14, 16, 18, 20, 22, $24,27,30,33,36,42,48,54$ et 60 mois. Chaque questionnaire est construit de la même façon et contient 30 items décrivant, dans un langage clair, simple et précis, des habiletés ou des comportements à différents niveaux du développement. Ces items sont divisés selon cinq domaines : communication, motricité globale, motricité fine, résolution de problèmes (cognitif) et habiletés individuelles ou sociales. Le répondant doit, pour les 30 items du questionnaire, cocher soit oui pour indiquer que l'enfant manifeste l'habileté spécifiée dans l'item, soit parfois pour indiquer une manifestation occasionnelle ou en émergence de la part del'enfant soit pas encore pour indiquer que l'enfant ne manifeste pas encore l'habileté, ou le comportement mentionné dans litem.

Les propriétés psychométriques de la version américaine (2e édition) ont été étudiées avec plus de 8000 questionnaires (Squires, Potter, \& Bricker, 1999). La fidélité test-retest, en comparant les scores de deux questionnaires complétés par le parent $(\mathrm{n}=112)$ à deux semaines d'intervalle, était de $94 \%$. La fiabilité inter-juges, en comparant la catégorie dans laquelle l'enfant se retrouvait en se basant sur le questionnaire rempli par le parent $(\mathrm{n}=112)$ et un intervenant $(\mathrm{n}=112)$, étai également de 94\%. La validité de construit considérée comme le pourcentage d'accord entre la catégorie à risque à l'ASQ et une évaluation standardisée varie entre $76 \%$ pour le questionnaire 4 mois et $91 \%$ pour le questionnaire 36 mois pour une moyenne de $88 \%$. La sensibilité (la capacité de l'ASQ à détecter les retards de développement) varie entre $51 \%$ pour le questionnaire 4 mois et $90 \%$ pour le questionnaire 36 mois pour une moyenne de $76 \%$.
La spécificité, la capacité pour l'ASQ d'identifier correctement les enfants ayant un développement typique varie entre $81 \%$ pour le questionnaire 16 mois et $92 \%$ pour le questionnaire 36 mois pour une moyenne de $86 \%$. Lanalyse de la consistance interne révèle des alphas de Cronbach variant de 0,55 à 0,86 pour les questionnaires 36 et 48 mois (Squires et al., 1999). Pour ce qui est des questionnaires 42 et 54 mois, les alphas de Cronbach, variant de 0,66 à 0,83 , n'ont pu être obtenus quả partir d'une étude réalisée avec la 3e édition de loutil (Squires, Twombly, Bricker, \& Potter, 2009).

Dans le cadre de cette étude, quelques adaptations et modifications ont été apportées aux questionnaires. Par exemple, concernant la forme, le logo du Step by Step Child and Family Center et des graphiques culturels (peuple; objets culturels et traditionnels tels des drums et des plumes) ont été ajoutés en remplacement au logo et graphiques originaux. Pour ce qui est du contenu, dans les questionnaires 36 et 42 mois, au domaine communication, à litem « Ask your child to put the shoe on the table », il est suggéré d'utiliser un autre objet (pas seulement un soulier) car il est possible que le répondant trouve inacceptable de demander à l'enfant de mettre son soulier sur la table. Dans les questionnaires 48 et 54 mois, au domaine motricité fine, à litem « Does your child color mostly within the lines? », lintervenant fournit un dessin pour indiquer ce qui est acceptable ou non. Dans les questionnaires 33 et 42 mois, au domaine communication « What is your name? (first and last name) », si l'enfant dit son nom Mohawk, cette réponse est acceptée et l'enfant reçoit la cote maximum.

\section{Questionnaire sociodémographique des parents}

Un questionnaire sociodémographique, envoyé aux parents, comprend des questions sur l'origine ethnique, le revenu et la langue parlée à la maison. Ce questionnaire est accompagné du formulaire de consentement de Step by Step Child and Family Center.

\section{Déroulement}

Pour chaque enfant, les enseignantes complètent un ou deux questionnaires ASQ différents et ce, à un intervalle d'environ 12 mois. Un total de 277 questionnaires est complété par les enseignantes auprès d'enfants tout-venant dont 136 questionnaires pour les filles et 141 questionnaires pour les garçons. Pour ce qui est des groupes de discussion, les enseignantes ont été rencontrées à une reprise. Les rencontres ont une durée de trois heures. Les groupes de discussion visaient à donner l'opportunité de partager leurs vécus et leurs préoccupations. Les enseignantes avaient la tâche de noter, en cours d'expérimentation, leurs commentaires ou réflexions. 


\section{Résultats}

\section{Groupe de discussion}

L'équipe de recherche a réalisé un groupe de discussion avec les enseignantes afin de vérifier si les activités faites en classe permettaient de répondre aux questions de l'ASQ, la concordance de cet instrument avec la philosophie d'intervention du Step by Step Child and Family Center, l'accessibilité du matériel, sa pertinence du point de vue de la culture Mohawk et de Kahnawake et son utilité. Finalement, il est également exploré si les résultats de l'ASQ confirment l'impression générale de l'intervenant quant au développement d'un enfant.

Globalement, en ce qui concerne la passation de linstrument, les enseignantes ont mis en évidence qu'il peut être facilitant de voir l'enfant en individuel pour vérifier les habiletés ciblées dans l'ASQ. En effet, selon leur expérience, ladministration en classe peut être difficile étant donné le bruit et le fait que dautres enfants peuvent répondre à la place de celui pour lequel l'instrument est administré. Toutefois, ce mode dadministration demande une organisation différente des activités quotidiennes. Une autre difficulté à la passation en groupe est que pour une classe donnée, six questionnaires de l'ASQ peuvent être requis étant donné lâge différent des enfants. Finalement, les enseignantes se questionnent sur le soutien possible auprès de l'enfant lors de l'évaluation de l'habileté cotée. De plus, elles souhaiteraient parler en termes d'habiletés émergentes plutôt que le terme parfois. Des précisions du critère de réussite seraient également nécessaires pour ce qui est notamment du coloriage.

La concordance de l'instrument avec la philosophie d'intervention du Centre est vue positivement et les enseignantes recommandent clairement la poursuite de son utilisation. Pour ce qui est de l'accessibilité du matériel, le matériel est disponible pour la plupart des items sauf pour les casse-têtes de six morceaux et les escaliers. Les enseignantes recommandent la création d'une boîte de matériel pour chaque questionnaire afin de faciliter l'utilisation de l'outil.

En ce qui concerne la confirmation des perceptions de l'enseignante par rapport à lenfant, ces dernières déplorent que les comportements dérangeants ne soient pas mis en évidence. Elles suggèrent d'inclure des questions sur la sphère socio-émotionnelle/comportementale et sur les difficultés de communication. D'autre part, la section informations générales offre un bon portrait de l'enfant mais il est suggéré d'inclure une feuille de commentaires pour $\mathrm{y}$ inclure plus dinformations sur ce dernier.

En ce qui a trait à l'intégration de l'ASQ dans le processus régulier de travail au Centre, les enseignantes soulignent que son utilisation s'est réalisée trop tôt dans l'année à savoir, à un moment intense d'activités pour l'équipe de travail. À cet effet, il est suggéré d’avoir deux périodes de dépistage dans l’année. Il est également recommandé d’offrir aux plus jeunes un temps dacclimatation (environ six semaines).

Dans le cas de la concordance avec la culture Mohawk, peu d'items ont été jugés non pertinents à l'exception de ceux concernant le prénom. Il importe de bien déterminer quelle réponse est acceptable. À titre d'exemple, à la question « Lorsque vous demandez à votre enfant: Quel est ton nom?, répondil en disant sont prénom et son nom de famille? » (domaine communication), si l'enfant utilise le nom Mohawk, sa réponse ne devrait pas être pénalisée même si la réponse ne comporte qu'un seul mot.

Finalement, lors de la deuxième expérimentation de linstrument, cette dernière s'est avérée plus facile selon les enseignantes. Elles se sentaient mieux préparées et mieux organisées. Certaines ont développé des aides et des activités pour la passation. De plus, les discussions sur le développement de l'enfant ont été plus nombreuses.

\section{Consistance interne}

Seuls les résultats des questionnaires de $36,42,48$ et 54 mois sont présentés pour un total de 277 questionnaires complétés. Le nombre de données insuffisant pour les questionnaires de 4, 6, 8, $10,12,14,16,18,20,22,24,27,30,33$ et 60 mois inclusivement, n'a pas permis d'assurer des traitements statistiques fiables.

Tout d'abord, la consistance interne de chacun des quatre questionnaires (36, 42, 48 et 54 mois) est étudiée. Pour ce faire, les alphas de Cronbach pour chaque domaine et ce, pour les quatre questionnaires de même que les coefficients de corrélation (domaines-score global de développement), sont calculés.

Dans un premier temps, le Tableau 1 présente les alphas de Cronbach par domaine pour les quatre questionnaires ainsi que les alphas de Cronbach de la version américaine. Ces derniers sont présentés à titre indicatif afin de contraster les résultats. Les résultats pour chacun des quatre questionnaires de la présente étude révèlent des alphas de Cronbach (pour chaque domaine) qui séchelonnent de la façon suivante : pour le Q36 mois, la valeur des alphas est faible à bonne $(0,61$ à 0,74$)$ avec des valeurs de l'alpha insuffisantes pour les domaines « motricité globale » $(0,29)$ et « aptitudes individuelles ou sociales $»(0,41)$; pour le Q42 mois, la valeur des alphas est de faible, se situant entre 0,51 et 0,69 à l'exception des domaines « motricité globale et fine » où les valeurs de l'alpha sont insuffisantes avec un 0,38 .et 0,34 ; pour le Q48, la valeur des alphas est de bonne à élevée $(0,69$ à 0,83); le Q54 présente des valeurs bonnes à élevée se situant entre 0,68 et 0,90, avec une valeur de l'alpha insuffisante de 0,46 pour le domaine « aptitudes individuelles ou sociales ». 
First Peoples Child \& Family Review, Volume 5, Number 2, 2010

\begin{tabular}{|c|c|c|c|c|c|c|c|c|c|c|c|c|}
\hline \multirow[t]{2}{*}{ Questionnaires } & \multicolumn{2}{|c|}{$\mathrm{n}$} & \multicolumn{2}{|c|}{ Communication } & \multicolumn{2}{|c|}{ Motricité globale } & \multicolumn{2}{|c|}{ Motricité fine } & \multicolumn{2}{|c|}{$\begin{array}{l}\text { Résolution de } \\
\text { problèmes }\end{array}$} & \multicolumn{2}{|c|}{$\begin{array}{l}\text { Aptitudes } \\
\text { individuelles ou } \\
\text { sociales }\end{array}$} \\
\hline & $\mathrm{Ka}$ & ÉU & Ка & ÉU & $\mathrm{Ka}$ & ÉU & $\mathrm{Ka}$ & ÉU & Ka & ÉU & $\mathrm{Ka}$ & ÉU \\
\hline 36 mois $^{a}$ & 74 & 231 & 0,61 & 0,69 & 0,29 & 0,76 & 0,74 & 0,72 & 0,69 & 0,66 & 0,41 & 0,55 \\
\hline 42 mois $^{b}$ & 65 & 950 & 0,57 & 0,72 & 0,38 & 0,68 & 0,34 & 0,76 & 0,69 & 0,72 & 0,51 & 0,66 \\
\hline 48 mois $^{a}$ & 76 & 336 & 0,83 & 0,79 & 0,70 & 0,84 & 0,75 & 0,86 & 0,69 & 0,85 & 0,69 & 0,86 \\
\hline 54 mois $^{b}$ & 62 & 586 & 0,90 & 0,83 & 0,68 & 0,73 & 0,68 & 0,79 & 0,78 & 0,75 & 0,46 & 0,71 \\
\hline
\end{tabular}

La procédure selon laquelle le coefficient de Cronbach est recalculé suite à lextraction d'un item est exécutée pour les domaines présentant des alphas inférieurs à 0,60: « communication »(Q42), « motricité globale » (Q36 et Q42), « motricité fine » (Q42) et « aptitudes individuelles ou sociales (Q36, Q42 et Q54). Les résultats montrent des augmentations du coefficient de Cronbach. Particulièrement, lalpha du domaine « motricité globale » (Q36) passe de 0,29 à 0,42 et celui du domaine « motricité fine », (Q42) passe de 0,34 à 0,43. Toutefois, les augmentations des alphas ne sont pas suffisantes à l'exception du domaine « résolution de problèmes » $(\mathrm{Q} 42)$ dont lalpha passe de 0,51 à 0,65 lorsque litem 5 est extrait à savoir « Si vous demandez à votre enfant : Montre-moi le plus petit cercle?, désigne-t-il le plus petit? Posez-lui cette question sanslaider, c'est-à-dire sans faire de geste, pointer du doigt, ni regarder le plus petit cercle».

Dans un deuxième temps, le Tableau 2 présente les coefficients de corrélation de Pearson obtenus lors des analyses entre domaine et score total pour les questionnaires 36 mois, 42 mois, 48 mois et 54 mois. Les corrélations entre les quatre questionnaires et leurs domaines « communication », « motricité fine » et « résolution de problème » sont de faibles à élevées variant de 0,35 à 0,86 . Les corrélations de « motricité globale » $(0,35)$ du Q36 sont faibles. Celles des domaines du Q42 sont bonnes variant de 0,63 à 0,76. Pour le Q48, les corrélations sont de bonnes à très bonnes $(0,76$ à $0,86)$. Les corrélations du Q54 sont également très bonnes $(0,63$ à 0,85$)$. Les domaines « communication », « motricité fine », « résolution de problème » et « aptitudes individuelles et sociales » présentent de bonnes corrélations avec le score global chez les quatre questionnaires $(0,67$ à 0,86$)$. Par contre, les corrélations entre le domaine « motricité globale » et le score global chez les quatre questionnaires sont généralement moins puissantes variant entre 0,35 à 0,63 à lexception du questionnaire 48 mois où les corrélations sont de 0,76 .

\begin{tabular}{|l|c|c|c|c|c|c|}
\hline \multicolumn{2}{|c|}{ Tableau 2: Coefficients de corrélation de Pearson entre domaine et score global de chacun des cinq questionnaires pour l'échantillon de Kahnawake } \\
\hline \multicolumn{1}{|c|}{ Questionnaires } & $n$ & Communication & Motricité globale & Motricité fine & $\begin{array}{c}\text { Résolution de } \\
\text { problèmes }\end{array}$ & $\begin{array}{c}\text { Aptitudes } \\
\text { individuelles ou } \\
\text { sociales }\end{array}$ \\
\hline 36 mois & 74 & $0,79^{* * *}$ & $0,35^{* *}$ & $0,77^{* * *}$ & $0,68^{* * *}$ & $0,68^{* * *}$ \\
\hline 42 mois & 65 & $0,76^{* * *}$ & $0,63^{* * *}$ & $0,67^{* * *}$ & $0,75^{* * *}$ & $0,75^{* * *}$ \\
\hline 48 mois & 76 & $0,86^{* * *}$ & $0,76^{* * *}$ & $0,82^{* * *}$ & $0,82^{* * *}$ & $0,80^{* * *}$ \\
\hline 54 mois & 62 & $0,85^{* * *}$ & $0,63^{* * *}$ & $0,78^{* * *}$ & $0,80^{* * *}$ & $0,75^{* * *}$ \\
\hline${ }^{*} \mathrm{p}<, 01 ;{ }^{* *} \mathrm{p}<, 01 ;{ }^{* * *} \mathrm{p}<, 001$. & & & & \\
\hline
\end{tabular}

\section{Discussion}

Le but de létude était de vérifier si l'outil de dépistage Ages and Stages Questionnaires (Bricker \& Squires, 1999) pouvait être utile et approprié pour une utilisation par des éducateurs auprès d, enfants Mohawks. De façon générale, les résultats démontrent que lesphas provenant de léchantillon Mohawk offrent une consistance interne acceptable. Toutefois, sept résultats révèlent des alphas insuffisants (inférieurs à 0,60) à savoir les domaines « communication » $(\mathrm{Q} 42)$, « motricité globale $(\mathrm{Q} 36$ et Q42), « motricité fine » (Q42) et « aptitudes individuelles ou sociales » (Q36, Q42 et Q54). Létude de Dionne, Squires, Leclerc, Péloquin et McKinnon (2006) auprès d'un échantillon québécois révèlent des résultats semblables quant à la 
« communication » (Q42), la « motricité globale $\gg(\mathrm{Q} 42)$ et aux « aptitudes individuelles ou sociales » (Q42 et 54). Ceux de Dionne, McKinnon, Squires et Bricker (soumis) auprès des parents autochtones vont dans le même sens pour la « motricité globale $(\mathrm{Q} 36$ et $\mathrm{Q} 42)$ et les « aptitudes individuelles ou sociales » (Q36 et Q42). Quant à l'étude chinoise (Tsai, McClelland, Pratt, \& Squires, 2006), le Q36 présente également une faible consistance interne au domaine « aptitudes individuelles ou sociales ».

Les résultats de ces études révèlent une constance à savoir de faibles coefficients de consistance interne pour les domaines « motricité globale » et « aptitudes individuelles ou sociales ». Pour le domaine « aptitudes individuelles ou sociales », Tsai et al. (2006) constatent quil est possible que les items qui composent ce domaine évaluent deux domaines distincts plutôt qu'un seul. Bien que l'exercice d'extraire un item a été réalisé, l'augmentation de la valeur des alphas pour six des sept domaines est insuffisante. Quant au domaine « motricité globale», les résultats de Janson (2003) obtenu suite à l'utilisation de l'ASQ par les parents auprès d'enfants norvégiens, révèlent cette même tendance pour le questionnaire 42 mois. Spécifions que dans les deux situations le nombre de questionnaires utilisés est relativement petit en comparaison avec la version américaine. Quant aux corrélations entre domaine et score total, elles sont généralement bonnes. Une seule d'entre elles est plus faible soit le domaine «motricité globale $\gg(\mathrm{Q} 36)$.

Pour ce qui est du domaine « résolution de problèmes » (Q42), l'extraction de l'item 5 à savoir « Si vous demandez à votre enfant : Montre-moi le plus petit cercle ?, désigne-t-il le plus petit? Posez-lui cette question sans l'aider, c'est-à-dire sans faire de geste, pointer du doigt, ni regarder le plus petit cercle » permet au coefficient de passer de 0,51 à 0,65. Le retrait de cet item offre une amélioration appréciable de lalpha.

D'autre part, le groupe de discussion a permis aux enseignantes de partager leur appréciation et leur expérience d'utilisation de l'ASQ. Elles soulignent certaines difficultés à utiliser l'ASQ en classe en situation de groupe (dérangement causé par les autres enfants, organisation de la classe modifiée, utilisation de plusieurs questionnaires pour un même groupe). Elles suggèrent également l'utilisation du concept d'habiletés émergentes (plutôt que le score «parfois») et la création de boittes de matériel requis pour chaque questionnaire. Elles soulèvent que peu d'items sont non pertinents culturellement parlant à l'exception de litem sur le prénom de l'enfant dans le domaine « communication ».

Par ailleurs, comme les intervenantes de l'étude Dionne et al. (2006) au Québec et de Ball dans l'Ouest canadien (2006), les enseignantes du Step by Step Child and Family Center recommandent la poursuite de l'utilisation de loutil. Plus précisément, ces dernières suggèrent d'utiliser l'outil à deux périodes durant l'année et d’offrir aux plus jeunes une période d'acclimatation d'environ six semaines.

Finalement, en ce qui concerne la perception des enseignantes par rapport à l'enfant, on déplore que les comportements dérangeants ne soient pas mis en évidence. On suggère d'inclure des questions sur la sphère socio-émotionnelle/ comportementale et sur les difficultés de communication. Par ailleurs, soulignons l'existence d'un complément à l'instrument sur la dimension socio-émotionnelle à savoir l'Ages and Stages Questionnaires social-emotional (ASQ-SE) (Squires, Bricker, \& Twombly, 2002).

Cette étude comporte certaines limites notamment en ce qui concerne la taille de l'échantillon. L'expérience auprès de la communauté Mohawk de Kahnawake ne peut être généralisée à l'ensemble des peuples des Premières Nations au Québec et au Canada. En effet, cette communauté se situe tout près d'un grand centre urbain (Montréal). Au Québec, notamment, plusieurs communautés des Premières Nations occupent un territoire rural qui diffère en plusieurs aspects de celui auprès duquel cette étude a été réalisée. Cet état de fait n'enlève en rien à la pertinence de cette étude qui pourrait servir d'amorce à la création d'une plateforme offrant de linformation sur les types d'adaptations à apporter en fonction de la communauté qui utilise l'instrument.

\section{Conclusion}

Limportance d'utiliser des instruments d'évaluation adaptés aux caractéristiques et à la réalité des communautés autochtones est déjà bien identifiée. Cependant, comment s'assurer qu'un instrument soit approprié pour une utilisation auprès des peuples des Premières Nations. Ball (2006) souligne que la standardisation des instruments devrait minimalement inclure une représentativité des populations autochtones au niveau de léchantillon. Dans le cas de la version américaine de l'ASQ, léchantillon utilisé pour la standardisation incluait $15 \%$ d'enfants autochtones américains.

Une autre façon de s'assurer de l'adéquation d'un instrument pour une communauté donnée est la perception des utilisateurs del'instrument sur leur expérience et l'appréciation de ce dernier. Dans ce sens, en prenant appui sur cette expérimentation, nous pouvons mettre de l'avant que l'ASQ est un instrument approprié à la réalité de la communauté Mohawk de Kahnawake. Le très petit nombre ditems identifiés comme inappropriés ainsi que la préservation de la consistance interne de l'instrument soutiennent cette idée.

Cependant, il ne fait nul doute que l'intervenant qui utilise linstrument doit avoir une bonne connaissance de la culture de la communauté auprès de laquelle il l'utilise. Cette connaissance 


\section{First Peoples Child \& Family Review, Volume 5, Number 2, 2010}

lui permettra de faire une lecture et une analyse appropriée au contexte et émettre des réserves à considérer le cas échéant.

Finalement, tout en déplorant le peu d'instruments élaborés à partir de la perspective autochtone, cette situation ne devrait pas être une entrave au déploiement d'activités de dépistage des retards de développement dans le cadre des services destinés à la petite enfance.

\section{Références}

Ball J. (2006). Screening and assessment experiences review in First Nations early childhood programs in British Columbia. Prepared for First Nations and Inuit Health Branch.

Ball, J. (2010). Indigenous child project. Policy brief: Protocols for developmental monitoring, screening and assessment of aboriginal young children. Résumé récupéré le 2 février 2010 de http://www.ecdip.org/docs/pdf/IC\%20 Policy\%20Brief\%20format.pdf

BC Aboriginal Child Care Society (2003). Handbook of best practices in Aboriginal early childhood programs. British Columbia, Canada: BC Aboriginal Child Care Society.

Bricker, D., \& Squires, J. (1999). Ages and stages questionnaire (2e éd.). Baltimore: Paul H. Brookes.

CSSSPNQL (2008). Portrait régional de la situation en matière de besoins spéciaux pour la clientèle âgée entre 0-6 ans des Premières Nations du Québec. Québec: CSSSPNQL.

Dionne, C., McKinnon, S., Squires, J., \& Bricker, D. (soumis). ASQ in First Nation (Mohawk) in Canada: The psychometric properties of the tool and the adaptations. Manuscrit soumis pour publication.

Dionne, C., Squires, J., Leclerc, D., Péloquin, J., \& McKinnon, S. (2006). Cross-cultural comparison of a French Canadian and US developmental screening test. Developmental Disabilities Bulletin, 34(1-2), 43-56.

Dion-Stout, M., \& Jodoin, N. (2006). MCH Screening tool project: Final report. Prepared for The maternal \& child health program First Nations and Inuit health branch.

Hernandez, R.D. (1994). Reducing bias in the assessment of culturally and linguistically diverse populations. Journal of Educational Issues of Language Minority Students, 14, 269-300.

Janson, H. (2003). Norsk manualsupplement til Ages and stages questionnaires [Norwegian manual supplement for the Ages and Stages Questionnaires]. Oslo, Norway: Regionsenter for barne- og ungdomspsykiatri, Helseregion Øst/Sør.

Losardo, A., \& Notari-Syverson, A. (2001). Alternative approach to assessing young children. Baltimore: Paul H. Brooks Publishing Co.

Meisels, S. J. (1994). Designing meaningful measurements for early childhood. Dans B. L. Mallory et R. S. New (Éds), Diversity in early childhood education: A call for more inclusive theory, practice, and policy (pp. 219-245). New York: Cambridge University Press.

Mushquash, C.J., \& Bova, D.L. (2007). Cross-cultural assessment and measurement issues. Journal on Developmental Disabilities, 13(1), 53-65.

Palfrey,J. S., Singer,J. D., Walker, D. K., \& Butler,J.A. (1987). Early identification of children's special needs: A study in five metropolitan communities. Journal of Paediatrics, 111, 651-659.
Palmantier, M. (2005). Building a community of communities: Results \& discussion of the national roundtable on aboriginal ECD: What can research offer aboriginal head start? Winnipeg, Canada: Health Canada.

Royal Commission on Aboriginal Peoples (1996). Gathering strength: Report on the Royal Commission on Aboriginal Peoples, 13. Ottawa: Canada Communication Group Publishing.

Sandall, S., Hemmeter, M.L., Smith, B., \& McLean, M. (2005). DEC recommended practices in early intervention/early childhood special education (2e éd.). Longmont, CO: Sopris West.

Shonkoff, J.P., \& Philips, D.A. (Éds) (2000). From Neurons to neighbourhoods: The science of early child development. Washington: National Academy Press.

Squires, J., Nickel, R., \& Eisert, D. (1996). Early detection of developmental problems: Strategies for monitoring young children in the practice setting. Journal of Developmental and Behavioral Pediatrics, 17(6), 410-427.

Squires, J., Bricker, D., \& Twombly, L. (2002). The Ages and stages questionnaires: Social-emotional. A parent-completed, child-monitoring system for socialemotional behaviours. Baltimore: Brookes Publishing Co.

Squires, J., Potter, L., \& Bricker, D. (1999). The ASQ user's guide for the agencies and stages questionnaire: A parent-completed, child-monitoring system (2e éd.). Baltimore: Paul H. Brookes Publishing Co.

Squires, J., Twomblym E., Bricker, D., \& Potter, L. (2009). ASQ-3. User's Guide (3e éd.). Baltimore: Brookes Publishing Co.

Tsai, H.-L. A., McClelland, M. M., Pratt, C., \& Squires, J. (2006). Adaptation of the 36-month Ages and Stages Questionnaire in Taiwan: Results from a preliminary study. Journal of Early Intervention, 28(3), 213-225. 